\title{
Gut Microbiota and Sunitinib-Induced Diarrhea in Metastatic Renal Cell Carcinoma: A Pilot Study
}

\author{
Zhimin Su $\mathbb{D}^{1,2}$ \\ Linbin Lu' \\ Fuyu Chen ${ }^{3}$ \\ Jianhui Chen ${ }^{4}$ \\ Xiong Chen (iD) \\ 'Department of Oncology, 900th \\ Hospital of PLA, Fuzong Clinical Medical \\ College of Fujian Medical University, \\ Fuzhou, 350024, People's Republic of \\ China; ${ }^{2}$ Department of Medical \\ Oncology, Xiamen Branch, Zhongshan \\ Hospital, Fudan University, Xiamen, \\ 36I015, People's Republic of China; \\ ${ }^{3}$ Department of Substation Maintenance \\ Center, State Grid Quanzhou Electric \\ Power Supply Company, Quanzhou, \\ 362000, People's Republic of China; \\ ${ }^{4}$ Department of Urology, Fujian Medical \\ University Union Hospital, Fuzhou, \\ 350024, People's Republic of China
}

Correspondence: Xiong Chen Department of Oncology, 900th Hospital of PLA, Fuzong Clinical Medical College of Fujian Medical University, 156 North Xierhuan Road, Fuzhou, 350024, People's Republic of China

Email cxiongzpc@fjmu.edu.cn
Introduction: Sunitinib-induced diarrhea seriously affects the prognosis of patients with metastatic renal cell carcinoma (mRCC) and reduces their quality of life. We aim to explore and find the relationship between sunitinib-induced diarrhea and gut microbiota.

Methods: Feces were collected from $31 \mathrm{mRCC}$ patients receiving sunitinib treatment. To characterize the feces gut microbiome profiles of patients, the V3-V4 region of 16S rRNA sequencing was carried out in this study.

Results: Gut microbial diversity was decreased both in the severe diarrhea group and in the sunitinib-post group. The microbial composition with higher abundance of Bacteroides (mucus degrading bacteria) and lower abundance of Faecalibacterium, Oscillospira, Ruminococcaceae, Eubacterium and Coriobacteriaceae (butyrate-producing bacteria) were found in patients with diarrhea. Interestingly, the abundance of Actinobacteria was decreased in patients receiving sunitinib with severe diarrhea.

Conclusion: This study reported an association between gut microbiota and sunitinibinduced diarrhea. Defects of the butyrate-producing bacteria and the increase in Bacteroides may be the physiological basis of sunitinib-induced diarrhea.

Keywords: sunitinib-induced diarrhea, 16S rRNA sequencing, gut microbial diversity, butyrate-producing bacteria

\section{Introduction}

Kidney cancer, representing $3.7 \%$ of all recorded cancer cases, is one of the 10 most common cancers worldwide. ${ }^{1}$ The 5 -year survival rate of kidney cancer is $53 \%$ in locoregional phase (stage III) but decreased to $8 \%$ in metastatic phase, respectively. ${ }^{2}$ Vascular endothelial growth factor-tyrosine kinase inhibitors are approved for treatment in patients with metastatic renal cell carcinoma, including sunitinib, sorafenib, pazopanib and axitinib. ${ }^{3,4}$ Zhang Sh showed that sunitinib can significantly prolong the overall survival (OS) and progression-free survival (PFS) in patients with advanced renal cell carcinoma. In the meantime, the treatmentrelated side effects are also attracting increasing attention. ${ }^{5}$ Among the pivotal studies of first-line VEGF-TKIs, the cumulative incidence is $51 \%$ of all grades of diarrhea, and $10 \%$ of grade $3 / 4$ diarrhea ${ }^{6,7}$ High incidences of all grades of diarrhea were reported from patients on sunitinib (61\%), sorafenib (53\%), pazopanib (63\%) and axitinib (55\%), respectively. ${ }^{8}$ Diarrhea may jeopardize the patient's health and reduce the quality of life. Several consensus statements have been approved for the management of sunitinib's toxicities, but the recommendations offered therein are merely supported by clinical experience. ${ }^{9}$ Thus, how to prevent and manage 
diarrhea is of vital importance since dose reductions and treatment discontinuations may significantly affect the final outcome.

Gut microbiota are located in the intestinal epithelial mucosa. Their physiological functions include maintaining the local barrier homeostasis, affecting the metabolism, inflammation, immune system and other functions of the human body. ${ }^{10}$ The gut microbiota regulates barrier functions and mucosal immune homeostasis by interacting with epithelial and stromal intestinal cells. ${ }^{1-13}$ Janelsins $\mathrm{BM}$ and Park $\mathrm{JH}$ both reported that the gut microbiota is involved in inflammatory immunity via lipopolysaccharide (LPS) or butyrate formation in colon cells. ${ }^{14-16}$ It has been commonly assumed that sunitinib may cause direct damage to colonic mucosa. Latterly this assumption is further supported by the data which shows the addition of VEGF inhibitors significantly reduced the capillaries network in intestinal villi. ${ }^{17}$ In recent years, several studies indicated that a mixture of pancreatic, neurologic and vascular bowel dysfunction with potential over-growth of gut microbes may account for sunitinib-induced diarrhea. ${ }^{8}$ In recent years, immune checkpoint inhibitors (ICIs) and tyrosine kinase inhibitors (TKIs) have been widely used in patients with primary mRCC. ${ }^{3,18}$ A meta-analysis suggested an increased risk of all-grade diarrhea, grade 3-4 diarrhea and grade 3-4 decreased appetite in patients treated with immuno-TKI combinations. ${ }^{19}$ In addition, Pal SK has demonstrated that intestinal flora in patients with mRCC has changed after VEGF-TKIs treatment. ${ }^{20}$ However, the composition and diversity of the gut microbiota and their correlation with prognosis remains unclear.

Our study aims to assess the relationship between sunitinib-induced diarrhea and gut microbiota initially explore the correlation between gut microbiota and gastrointestinal toxicity of sunitinib and provide a new strategy for prevention, diagnosis and treatment of sunitinibinduced diarrhea.

\section{Materials and Methods}

\section{Patient Selection}

The main criteria for this study were renal cell carcinoma with distant metastasis, no diabetes, hypertension or digestive tract disease, and currently receiving sunitinib. Each patient was treated with sunitinib for at least 2 weeks. Patients who had participated in other studies took probiotics within 8 weeks or combined treatment were excluded in this study. Written informed consent was obtained from each patient. This study was carried out in accordance with the principles of the Helsinki declaration. Participants' demographics, clinicopathological data and eating habits were collected from hospital electronic medical records and follow-up phone inquiries.

\section{Specimen Collection}

A standard operating procedure was used by all the patients. A total of 31 patients were enrolled in this study. Finally, 33 fecal samples were collected (31 samples were collected after sunitinib treatment, while 2 samples were collected before sunitinib treatment). The fecal samples collected directly by participants froze at $-80^{\circ} \mathrm{C}$ within 24 hours of collection. The microbiota of all fecal samples was assessed by the V3-V4 region of $16 \mathrm{~S}$ rRNA sequencing.

\section{Ethics Approval and Consent to Participate}

The Ethics Committee of the 900th Hospital of PLA Biomedical Research Department provided ethical approval, and informed consent for collecting and preserving samples and details was obtained from each patient.

\section{Diarrhea Characterization}

Patients were diagnosed as having grade 1-4 diarrhea or no diarrhea by using Common Toxicity Criteria for Adverse Events (CTCAE;4. 0). Based on the severity of diarrhea, the patients were divided into a mild diarrhea group (grade 1-2 diarrhea) and severe diarrhea group (grade 3-4 diarrhea). Each patient with or without diarrhea submitted fecal samples, and together reported the symptoms related to diarrhea at the same time.

\section{Extraction of Genome DNA and PCR Products}

Total genome DNA was extracted using the standard CTAB/SDS method. DNA concentration and purity were detected by $1 \%$ agarose electrophoresis. DNA was diluted to $1 \mathrm{ng} / \mu \mathrm{L}$ with sterile water for sequencing. Primers used in this study: 16S V4-V5:515F-907R, 18S V9:1380F1510R, ITS1: ITS1F- ITS2R. 16S/18S rRNA fragments were amplified using the specific primer. All PCR reactions were carried out in $30 \mu \mathrm{L}$ reactions with $15 \mu \mathrm{L}$ of Phusion High-Fidelity PCR Master Mix (New England Biolabs); $0.2 \mu \mathrm{M}$ of forward and reverse primers, and about $10 \mathrm{ng}$ template DNA. Thermal cycling reaction 
protocol: initial denaturation at $98^{\circ} \mathrm{C}$ for $1 \mathrm{~min}$, followed by 30 cycles of denaturation at $98^{\circ} \mathrm{C}$ for $10 \mathrm{~s}$, annealing at $50^{\circ} \mathrm{C}$ for $30 \mathrm{~s}$, and elongation at $72^{\circ} \mathrm{C}$ for $60 \mathrm{~s}$, and final extension at $72^{\circ} \mathrm{C}$ for $5 \mathrm{~min}$. PCR products were mixed with $1 \mathrm{X}$ loading buffer (contained SYB green) and loaded on $2 \%$ agarose gel for electrophoresis detection. Samples between 400 and 450 bp length with a bright main strip were collected for further experiments. PCR products were mixed in equidensity ratios and then purified with GeneJET Gel Extraction Kit (Thermo Scientific).

\section{Library Preparation and Multiplex Sequencing}

Sequencing libraries were constructed using NEB Next ${ }^{\mathbb{R}}$ Ultra $^{\mathrm{TM}}$ DNA Library Prep Kit for Illumina (NEB, USA) following standard protocols and index codes were added for sequencing. The library quality was tested on the Qubit@2.0 Fluorometer (Thermo Scientific) and Agilent Bioanalyzer 2100 system. The library was sequenced on an Illumina MiSeq platform, and about 250 bp/300 bp length of paired-end reads were generated and collected.

All fecal samples from patients were sequenced using V3-4 regions. 16S rRNA genes were amplified using a specific primer with a barcode. The following PCR primers were used to amplify $16 \mathrm{~S}$ V3-V4 region: $341 \mathrm{~F}$ 806R. Software QIIME was used to analyze the Illumina sequencing reads generated for the V3-V4 region of $16 \mathrm{~S}$ rRNA. $^{21}$

\section{OUT Cluster and Species Annotation}

Sequence analysis was performed using the UPARSE software package using the UPARSE-OTU and UPARSEOTUref algorithms. ${ }^{22}$ Inhouse Perl scripts were used to analyze alpha (within samples) and beta (among samples) diversity. We pick a representative sequence for each OTU and use the RDP classifier to annotate taxonomic information for each representative sequence. The obtained sequences with $\geq 97 \%$ similarity were assigned to the same operational taxonomic units (OTUs). The diversity and taxonomic analysis of gut microbiota were determined by sampling-based OTUs analysis and presented by Shannon index and Rarefaction curves. ${ }^{23,24}$ The Rank-abundance distribution curve can be used to explain two aspects of diversity, namely species richness and species evenness. ${ }^{25}$ In horizontal direction, the abundance of species is reflected by the width of the curve. The higher the abundance of species, the larger the range of the curve on the horizontal axis. The shape of the curve (smoothness degree) reflects the average of species in the sample. The smoother the curve is, the more uniform the distribution of species is. Bacterial diversity and taxonomic analysis were determined by sampling-based OTUs analysis. All OTUs annotations are listed in the online Supplementary Table S1.

\section{PCoA Analysis and Community Distribution}

Unweighted unifrac distance was used for Principal Coordinate Analysis (PCoA) in this study. PCoA can help to obtain principal coordinates and visualize them from complex, multidimensional data by taking a transformation from a distance matrix to a new set of orthogonal axes. ${ }^{26}$ Using PCoA, the maximum variation factor was demonstrated by the first principal coordinate, and the second maximum factor by the second principal coordinate, respectively. Graphical representations of the relative abundance of microbes from phylum to species can be visualized using Krona chart. ${ }^{27}$ To validate differences in the abundances of individual taxonomy between the two groups, metastats software was applied in this study.

\section{Difference Analysis}

Linear discriminant analysis (LDA analysis) was used for the quantitative analysis of biomarkers within different groups. ${ }^{28}$ This LDA analysis was designed to analyze data where the number of species is much higher than the number of samples and to provide biological elucidation to establish statistical significance, biological consistency, and effect size estimation of predicted biomarkers.

\section{Statistical Analyses}

Paired Wilcoxon rank-sum tests were conducted to detect differences in clinical parameters between the two groups. A $\mathrm{P}$ value or an adjusted $\mathrm{P}$ value of less than 0.05 was considered statistically significant. Statistical analyses were performed using SPSS V. 20.0 (SPSS, Chicago, Illinois, USA).

\section{Results}

\section{Baseline Characteristics of Patients}

A total number of 31 patients were consented and enrolled in this study. Most of the patients were male (77.4\%). Of these patients, 20 of them had sunitinib-induced diarrhea, while the other 11 did not. The median age at diagnosis was 59 . There was no difference between the two groups in relation to gender, age and BMI score (Table 1). The most frequent 
metastatic tissues (in descending order) were lung, bone and lymph node metastasis, most of which had higher KPS scores. Most of the patients had intermediate IMDC risk in these two groups, only four patients faced with severe risk (Table 1). Each patient continued to receive sunitinib treatment lasting for an average of 19.1 months (ranging from 1.5 to 112 ) before collecting fecal sample.

\section{Gut Microbial Diversity}

The species accumulation curves became flatter as the sample size increased, indicating that the sample size of this study was sufficient (Figure 1A). The estimated OTUs richness, which

Table I Baseline Characteristics of Patients

\begin{tabular}{|c|c|c|c|}
\hline Characteristic & Diarrhea (\%) & $\begin{array}{l}\text { Non- } \\
\text { Diarrhea } \\
\text { (\%) }\end{array}$ & $\begin{array}{l}P \text { values } \\
\text { (Diarrhea vs } \\
\text { Non) }\end{array}$ \\
\hline & $N=20$ & $N=||$ & \\
\hline $\begin{array}{l}\text { Age }(y) \\
\text { Median } \\
\text { (range) } \\
\quad 18-64 \\
\geq 65\end{array}$ & $\begin{array}{l}59.5(36-72) \\
14(70) \\
6(30)\end{array}$ & $\begin{array}{l}56(47-75) \\
9(82) \\
2(18)\end{array}$ & $p=0.676$ \\
\hline $\begin{array}{l}\text { Sex } \\
\qquad \text { Male } \\
\text { Female }\end{array}$ & $\begin{array}{l}15(75) \\
5(25)\end{array}$ & $\begin{array}{l}9(82) \\
2(18)\end{array}$ & $p=1.000$ \\
\hline $\begin{array}{l}\text { BMl, kg/m2 } \\
\quad<18.5 \\
\quad 18.5-24.9 \\
\geq 25\end{array}$ & $\begin{array}{l}3(15) \\
10(50) \\
7(35)\end{array}$ & $\begin{array}{l}\text { I (9) } \\
5(45) \\
5(45)\end{array}$ & $p=0.809$ \\
\hline $\begin{array}{l}\text { KPS score } \\
\quad<80 \\
\geq 80\end{array}$ & $\begin{array}{l}0(0) \\
20(100)\end{array}$ & $\begin{array}{l}4(45) \\
7(27)\end{array}$ & $p=0.010$ \\
\hline Metastatic site & & & $p=0.057$ \\
\hline $\begin{array}{l}\text { Lung } \\
\text { Bone } \\
\text { Lymph nodes } \\
\text { others }\end{array}$ & $\begin{array}{l}\text { I3 (65) } \\
6(30) \\
5(25) \\
I(5)\end{array}$ & $\begin{array}{l}2(18) \\
5(45) \\
1(9) \\
3(27)\end{array}$ & \\
\hline $\begin{array}{l}\text { IMDC risk } \\
\text { group } \\
\text { Intermediate } \\
\text { poor }\end{array}$ & $\begin{array}{l}19(80) \\
1(5)\end{array}$ & $\begin{array}{l}8(82) \\
3(0)\end{array}$ & $p=0.115$ \\
\hline \multicolumn{2}{|c|}{ Number of prior therapies } & & $p=0.739$ \\
\hline $\begin{array}{l}0 \\
1 \\
2\end{array}$ & $\begin{array}{l}\text { I5 (75) } \\
4(20) \\
I(5)\end{array}$ & $\begin{array}{l}9(82) \\
2(18) \\
0(0)\end{array}$ & \\
\hline
\end{tabular}

significantly decreased from the non-diarrhea group to the diarrhoea group, was basically close to the saturation level in each group by rarefaction analysis (Figure 1B), especially in the severe group (Figure 1C). The same result could be obtained from the rank-abundance distribution analysis (Figure 1D). Similarly, the rank-abundance distribution decreased in the severe group compared with the mild group (Figure 1E). In addition, Shannon analysis showed that gut microbial diversity in the diarrhea group was lower than that in the non-diarrhea group, but the results were not statistically significant $(\mathrm{p}=0.168)$ (Figure $1 \mathrm{~F})$; however, gut microbial diversity was significantly higher in the severe group than that in the mild group $(\mathrm{p}=0.048)$ (Figure $1 \mathrm{G})$. Moreover, beta diversity was calculated with unweighted UniFrac using PCoA, indicating a symmetrical distribution of fecal microbiome among all the samples (Figure $1 \mathrm{H}$ ).

\section{Microbial Community Composition}

Phylogenetic profiles of fecal microbiome phylotypes with a median relative abundance, which was larger than $0.01 \%$ of total abundance were included for comparison. The average composition of microbiome at the phylum and genus levels were shown in Figure 2A. Bacterial phyla of Firmicutes, Bacteroidetes, and Proteobacteria were the three predominant classes in non-diarrhea groups and Bacterial phyla of Firmicutes, Bacteroidetes, Proteobacteria and Fusobacteria were the four predominant classes in non-diarrhea groups at phylum level, accounting for up to $90 \%$ of total sequences on average (Figure $2 \mathrm{~B}$ ). The microbial community distribution of the two groups at genus level was shown in the online Supplementary Figure S1.

\section{The Change of Gut Microbiota in the Diarrhea Group}

Notably, the abundance of Tenericutes significantly decreased in the diarrhea group $(\mathrm{p}=0.037)$ (Figure 3A). At the genus level, 7 genera of bacteria, including Faecalibacterium, Oscillospira, Ruminococcaceae, Eubacterium, Coriobacteriaceae, Ralstonia and Lachnospiraceae, their abundances in gut microbiota significantly decreased (All $\mathrm{p}<0.05$, Figure 3B-J). Surprisingly, the abundance of Actinobacteria was found to be increased in the severe group $(\mathrm{p}=0.041)$ (Figure $3 \mathrm{~K})$.

\section{Difference Analysis}

To validate the types of bacteria that had changed, LDA analysis was carried out in this study. The cladogram showed 
A

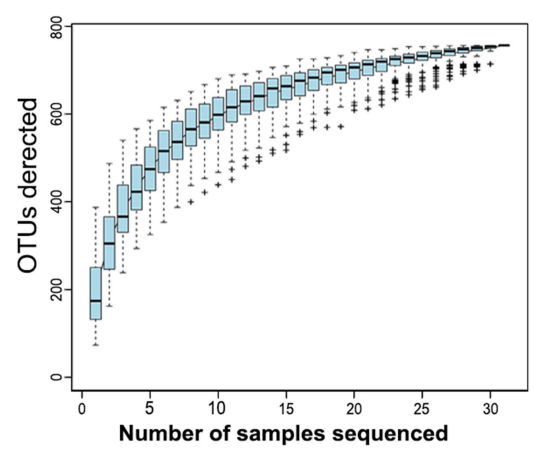

B



C

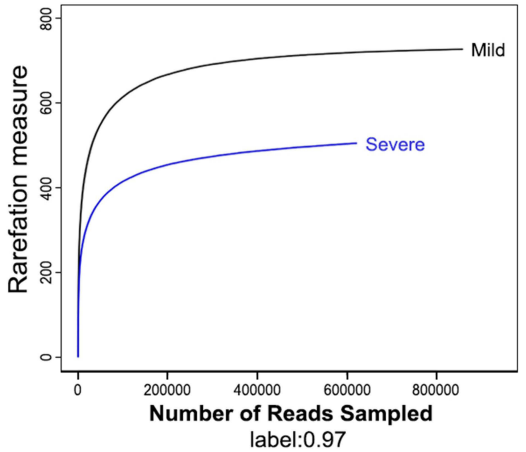

D

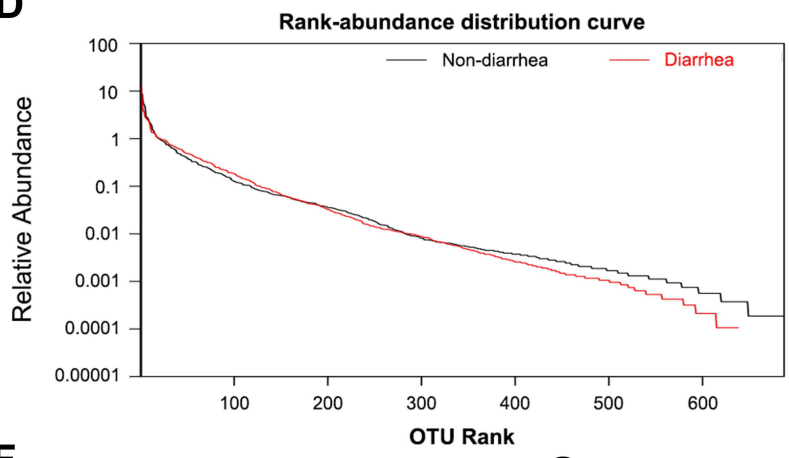

F

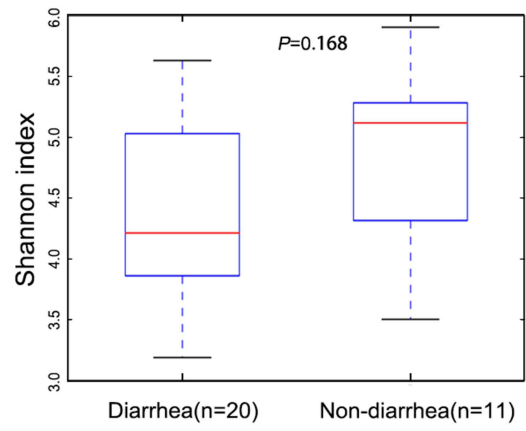

E

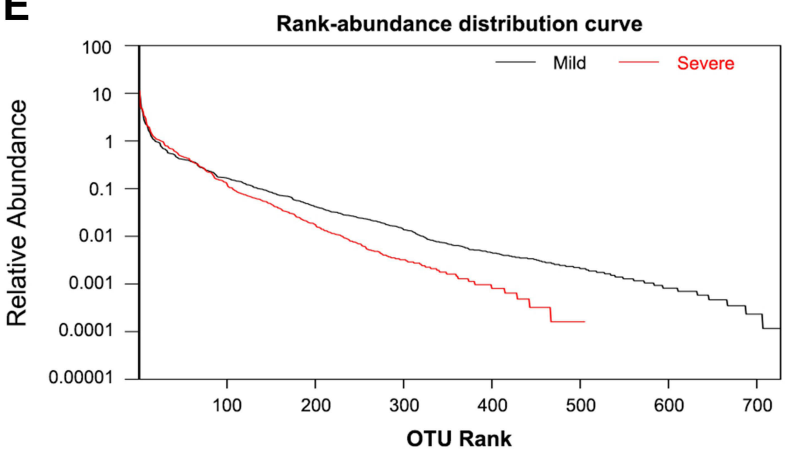

H

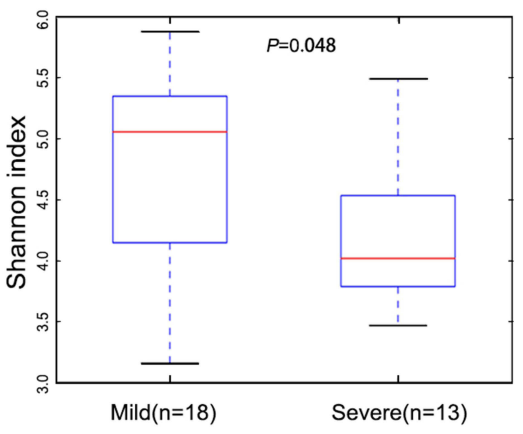

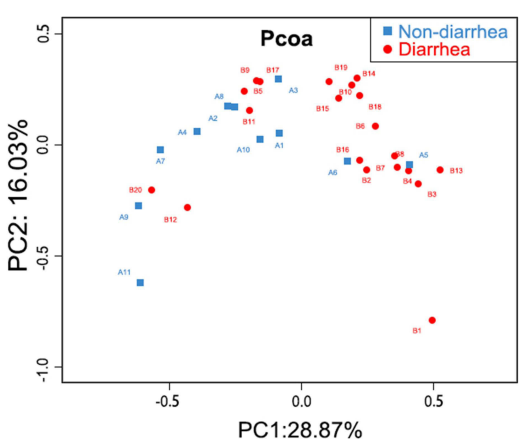

Figure I Gut microbiota diversity in patients $(n=31)$. (A) Species accumulation curves became flatter as the sample size increased, indicating that the sample size of this study is adequate. (B) The estimated OTUs richness was significantly decreased from the non-diarrhea group ( $\mathrm{n}=\mathrm{I}$ I) to the diarrhea group ( $\mathrm{n}=20$ ). (C) The estimated OTUs richness was significantly decreased in the severe group $(n=13)$. (D) Rank-abundance distribution curves analysis showed that the relative abundance of the diarrhea group was lower than that of the non-diarrhea group. (E) The relative abundance of the severe group decreased compared with the mild group. (F) Gut microbial diversity in the diarrhea group was lower than that in the non-diarrhea group by Shannon analysis. (G) Gut microbial diversity was significantly higher in the severe group than that of in the mild group $(p=0.048)$. (H) PCoA analysis indicated a symmetrical distribution of fecal microbial community among all the samples.

Abbreviations: OTUs, Operational Taxonomic Units; Boxes represent the interquartile ranges, lines inside the boxes denote medians; PCoA, principal coordinates analysis.

that the bacterial abundances of Prevotellaceae, Christensenellaceae, Family XIII, Ruminococcaceae and Burkholderiaceae significantly decreased in the diarrhea group versus non-diarrhea group (all $\mathrm{p}<0.05$ ), while the abundances of Pasteurellaceae increased in the diarrhea group (Figure 4A). LDA scores were obtained by LDA analysis for the significant microbial communities in the two groups. The higher the scores are, the more significant the difference in microbial abundance is between the two groups. In the nondiarrhea group, Ruminococcaceae had the highest score, while Pasteurellaceae had the lowest score $(\mathrm{p}=0.0352$, 0.0408 , respectively) (Figure 4B), similar to the cladogram. 


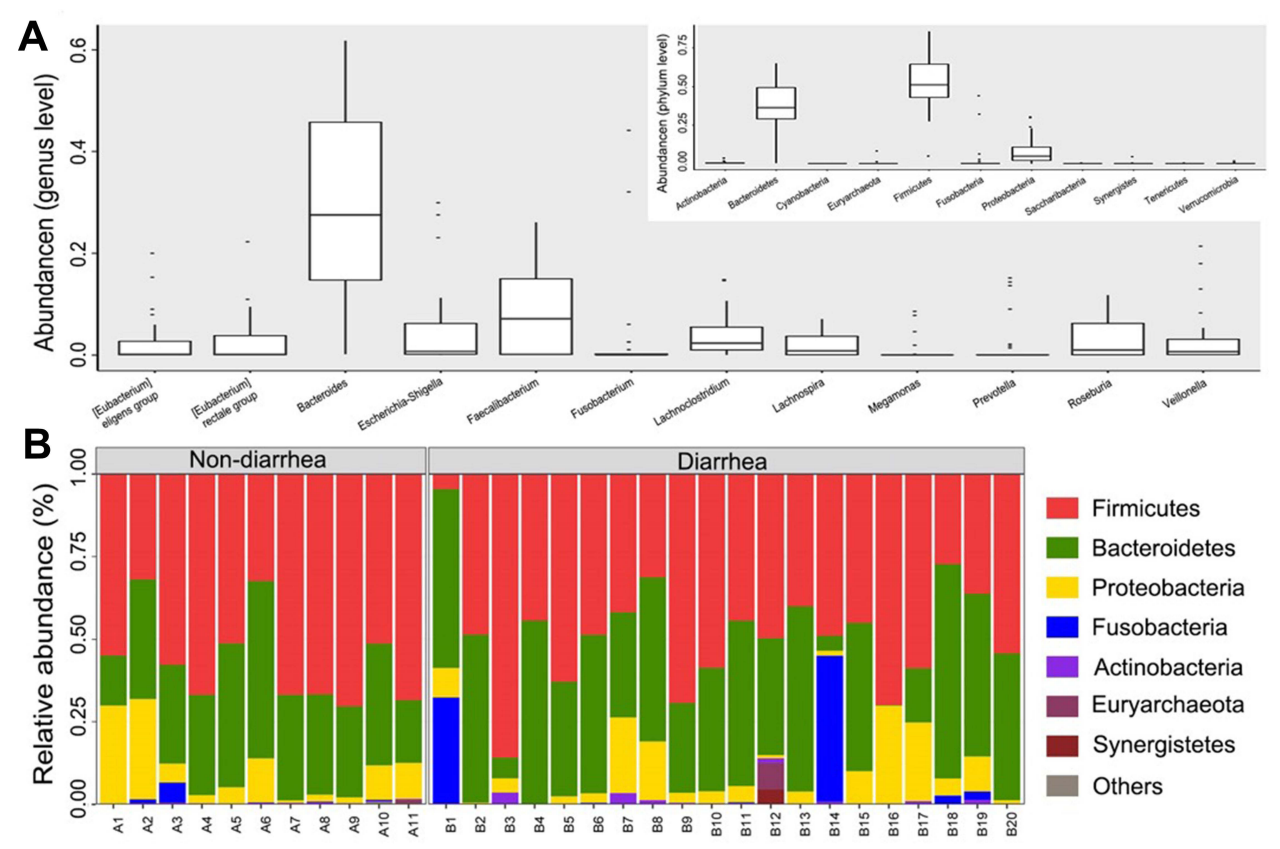

Figure 2 The composition of the microbial community and the predominant classes in two groups. (A) Composition of fecal microbiota at the phylum level and genus level. (B) The predominant classes of microbes in two groups at phylum level. The part of the abundance less than $1 \%$ was merged into others when plotting.

\section{Bacteroides Decreased in Patients with Sunitinib}

To further elucidate whether the sunitinib had affected the intestinal flora of an individual patient or the composition of fecal microbiota was preexisted, we additionally collected and analyzed two stool samples of patients before therapy. Rank-abundance distribution curves analysis showed that the microbial diversity decreased after treatment compared with that of previous treatment (Figure 5A). It is noteworthy that the abundance of Bacteroides decreased in sunitinib-post group (Figure 5B). This finding further revealed that patients with mRCC after taking sunitinib have an altered microbiota profile, which may relate to the vulnerability causing diarrhea. The specific LDA score value is shown in the online Supplementary Table S2.

\section{Discussion}

Notably, our study is the first to report changes in gut microbiota in patients with $\mathrm{mRCC}$ before and after treatment. Until now, limited studies indicated that there was a direct association between gut microbiota and sunitinibinduced diarrhea. Considering the incidence of gastrointestinal toxicity associated with VEGF-TKIs, we proposed that gut microbiota may be associated with the occurrence of diarrhea. ${ }^{29}$ In this study, we sequenced the total microbiome of fecal samples from 31 individuals with mRCC. We found that compared with the non-diarrhea group, the gut microbial diversity decreased in the severe diarrhea group. Moreover, the microbial diversity also decreased after taking sunitinib compared with that of before treatment. Therefore, the decline of bacterial diversity implies that greater richness or diversity in the gut microbiota community is a signal of a healthy gut microbiota in our cohort.

The alterations of gut microbiota in different chronic diseases such as inflammatory bowel diseases, T2D, atherosclerotic cardiovascular disease and chronic kidney disease are distinctive. ${ }^{30-33}$ Different diseases showed relatively characteristic microbial composition and diversity. ${ }^{34}$ Here, in patients with sunitinib-induced diarrhea, the richness of genera Faecalibacterium, Oscillospira, Ruminococcaceae, Eubacterium and Coriobacteriaceae, which belong to butyrate-producing bacterial families, decreased. ${ }^{35}$ As a major energy source for mucosal cells, butyrates are considered as important regulators for gene expression, inflammation, differentiation and apoptosis in host cells and appear to play pivotal roles in bacterial energy metabolism and gut health. ${ }^{36}$ Qin $\mathrm{J}$ had demonstrated that the abundances of butyrate- 




E
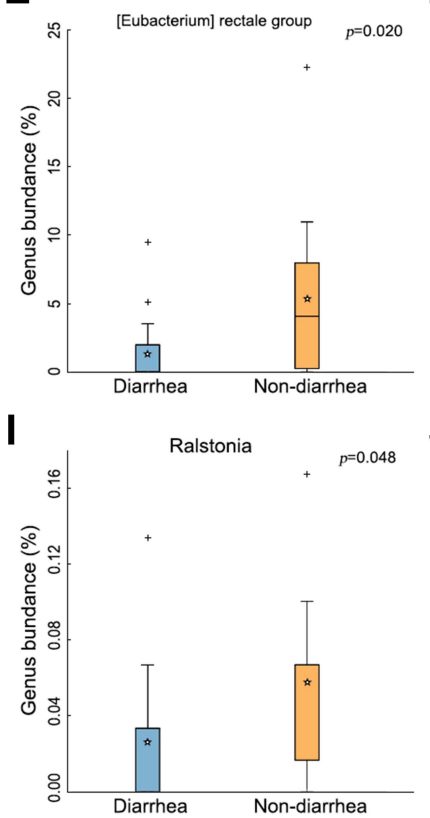

B C

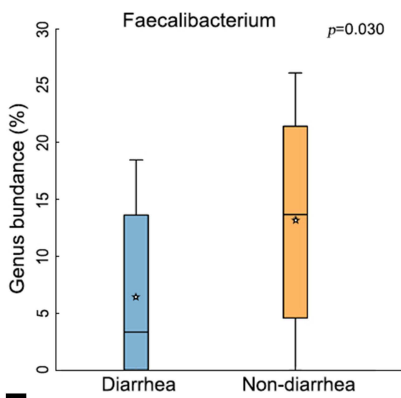

F
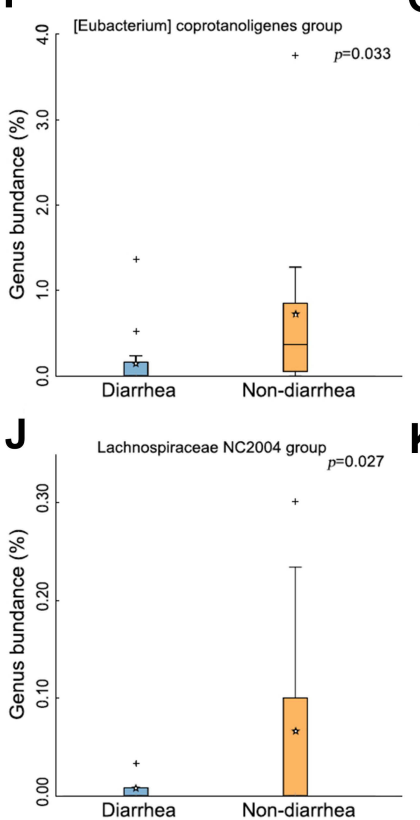

G
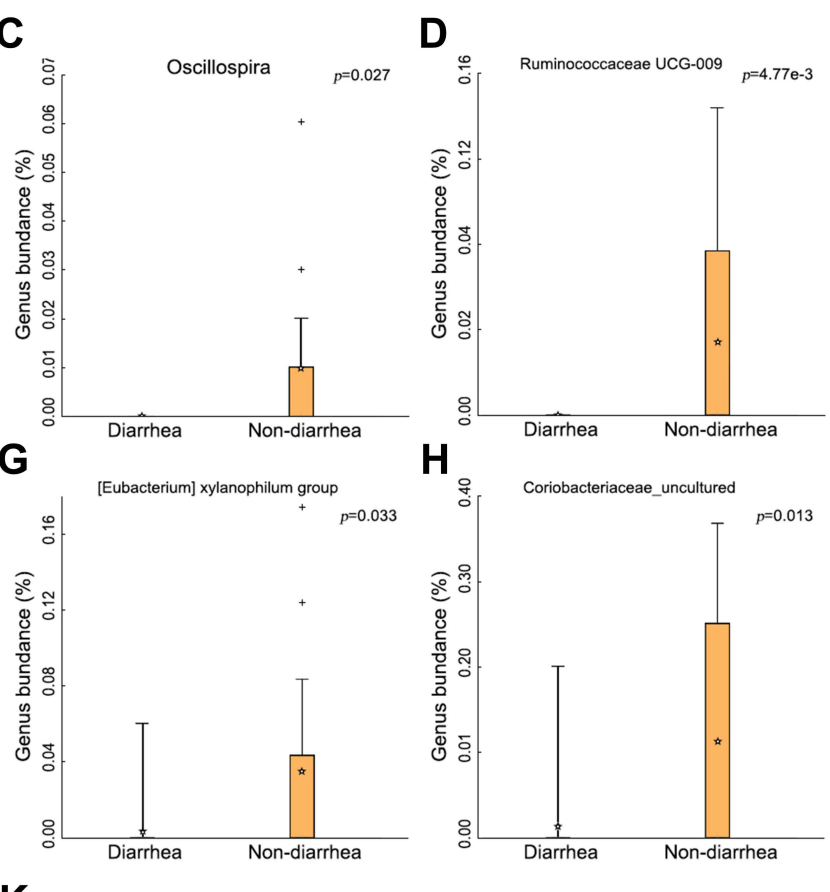

H

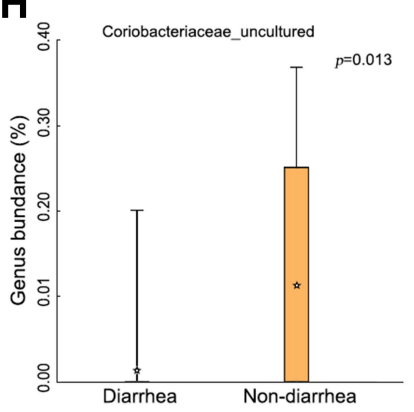

Figure 3 The change of gut microbiota in the diarrhea group. (A) Tenericutes significantly decreased in the diarrhea group ( $\mathrm{p}=0.037)$. (B-J) Types of bacteria reduced in diarrhea group. (K) Less Actinobacteria was found in the severe group ( $\mathrm{p}=0.04 \mathrm{I})$. The box presented $95 \% \mathrm{Cls}$; the line inside denotes the median, and the symbol "+" denotes the mean value.

producing microbes in patients with $\mathrm{T} 2 \mathrm{D}$ were significantly decreased. ${ }^{33}$ Moreover, the richness of Bacteroides was observed to be increased in patients with sunitinibinduced diarrhea but decreased in the sunitinib-post group. Therefore, the association between Bacteroides and the severe diarrhea group indicated the overgrowth of various harmful bacteria or archaea in patients with sunitinibinduced diarrhea. Bacteroides belong to mucus degrading bacteria. Mucus degradation led to increased susceptibility to the mucosal pathogen Citrobacter rodentium, resulting in a "leaky gut" condition and colitis, which is a risk factor for diarrhea. ${ }^{37}$ A preclinical study showed that the relative increase in Bacteroides has a positive effect on chemotherapy-induced diarrhea in the mucositis rat model. ${ }^{38}$ Interestingly, we found that the decrease of Actinobacteria in patients receiving sunitinib with severe diarrhea was likely consistent with the decrease of
Akkermansia. Akkermansia muciniphila is a Gramnegative anaerobic microbe that uses secreted mucins as carbon and nitrogen source. Desirable modulation of Akkermansia muciniphila can provide health benefits by affecting microbiome-related metabolic profiles. ${ }^{39}$ The administration of Akkermansia muciniphila increased the intestinal levels of endocannabinoids that control inflammation, the gut barrier, and gut peptide secretion. Briefly, the decrease in butyrate-producing bacteria, the increase in mucus degrading bacteria and the potential decrease in Akkermansia muciniphila may promote intestinal mucosal destruction, thereby contributing to sunitinib-induced diarrhea. In the past few years, ICIs plus TKIs have made a major breakthrough in the treatment of $\mathrm{mRCC}$ patients. $^{3,18,40}$ Comparison with the sunitinib group, a higher proportion of grade 3-4 diarrhea and grade 3-4 decreased appetite occurred in patients treated with 

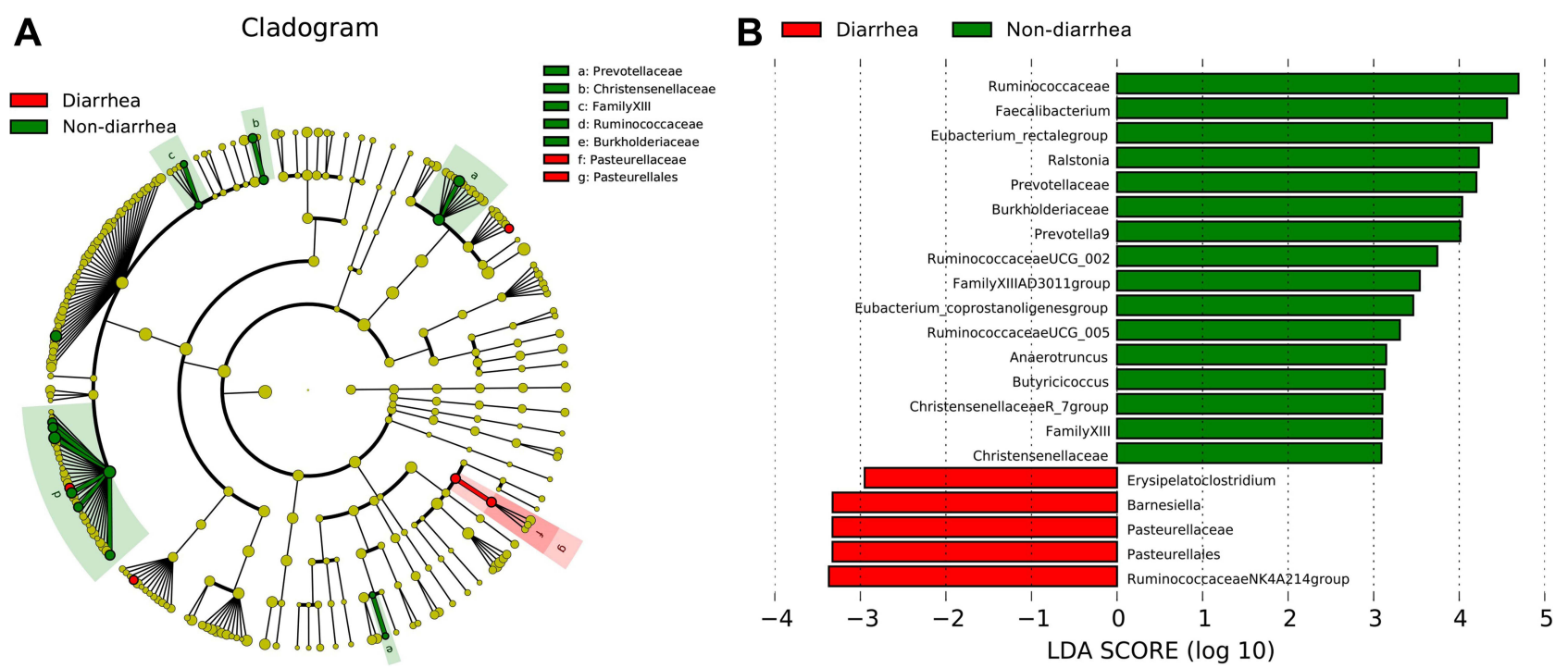

Figure 4 The change of bacteria displayed by LDA analysis. (A) Cladogram. The red and green nodes in the branches represented the microbiota that play an important role in the corresponding groups, respectively, while the yellow nodes represented the microbiota that do not play an important role in the two groups. (B) The LDA scores obtained by LDA analysis for the significant microbial communities in the two groups. The red and green regions indicated the abundance of different microbes.
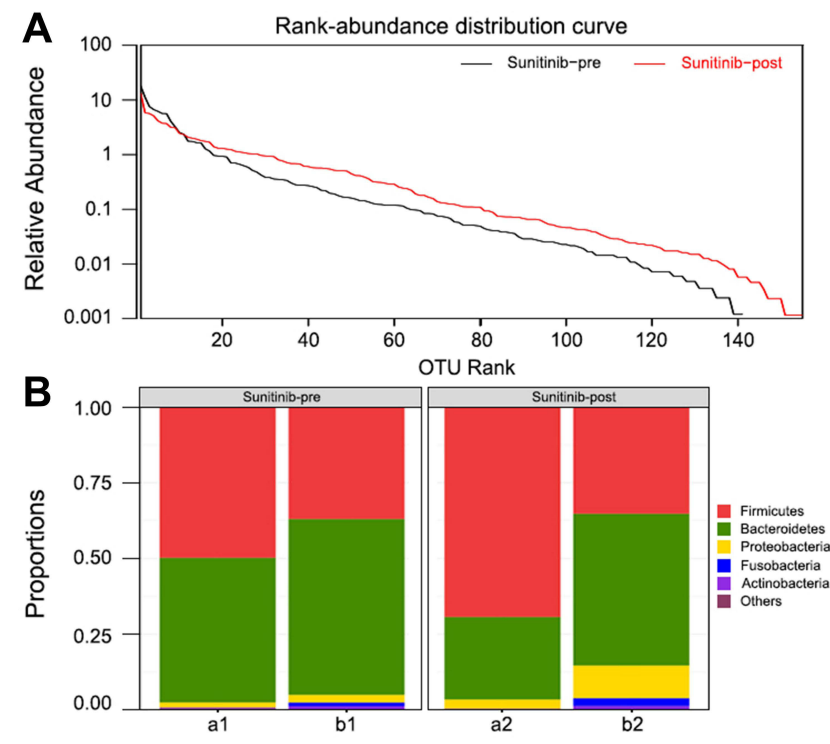

Figure 5 Bacteroides in patients with sunitinib. (A) Rank-abundance distribution curve. The bacterial diversity increased after sunitinib treatment. (B) Microbial community composition of patients before and after treatment. The abundance of Bacteroides was decreased in patients with sunitinib.

immuno-TKI combinations in a meta-analysis. ${ }^{19}$ There remains a critical need to better predict treatment responses in patients with $\mathrm{mRCC}$ and to pre-emptively recognize patients at higher risk of treatment-attributable toxicities. Except the non-small-cell lung cancer, predictive biomarkers such as PD-L1 and tumor mutational burden have failed to consistently predict treatment outcomes. $^{3}$ In clinical practice, Salgia et al prospectively collected feces from $33 \mathrm{mRCC}$ patients with ICIs treatment, used whole-genome shotgun metagenomic sequencing to characterize microbiome, and identified specific bacteria such as Prevotella copri, Bifidobacterium adolescentis, and Faecalibacterium that were overrepresented in patients who experienced better outcomes. $^{41}$ These results implied that the above changed gut microbes may be prognostic factors and potential therapeutic targets to predict and manage sunitinibinduced diarrhea.

In recent years, preclinical data showed that Bifidobacterium Infant ameliorates chemotherapy-induced intestinal mucositis in colorectal cancer rats. ${ }^{42}$ Probiotics have been shown to prevent inflammatory bowel diseaserelated diarrhea, chemotherapy-induced diarrhea and other diseases. $^{43-45}$ Although, the use of probiotics has never been investigated in TKI patients, only individual patients report considerable benefits. In the future, probiotics fortified with butyrate-producing bacteria and Akkermansia muciniphila as a new therapeutic plan for sunitinibinduced diarrhea are promising. Meanwhile, elimination of potential pathogenic species such as Bacteroides may reduce side effects of sunitinib, thereby the prognosis of patients could be improved. At present, to beneficially shift the gut microbiome diversity and composition, relevant clinical studies using probiotics and fecal microbiota transplantation (FMT) (NCT03772899 and NCT03772899) have been carried out.

Although there were restrictions on the dietary factors in this study, the samples were heterogeneous in 
a number of respects. Gastrointestinal toxicity of sunitinib may be affected by other potential factors in the whole procedure of disease treatment. Also, the collections of fecal samples were not uniformly performed. Patients had received at least 2 weeks of sunitinib treatment before collecting samples, to provide time for diarrhea development. In further study, the stool will be collected at pre-determined time points as baseline, after 2 weeks and 6 months of treatment respectively. Previous work revealed that butyrate regulates the generation of colonic Tregs and dampens the effector T-cell responses. ${ }^{46}$ Blood samples will be collected additionally from patients and measured by flow cytometry. In addition, the expansion of sample size, the inclusion of healthy samples and the analysis of prognosis will be improved in the next study. Further validation of our work will be carried out to explore the potential mechanisms by which the gut microbiota affects sunitinibinduced diarrhea.

\section{Conclusion}

This study showed that the severity of sunitinib-induced diarrhea was found to be inversely correlated with gut microbiota diversity and butyrate-producing bacteria, but positively correlated with Bacteroides. Compositional changes in gut microbiota may be a prognostic factor and a potential therapeutic target to prevent and manage sunitinib-induced diarrhea. Probiotics fortified with butyrateproducing bacteria and Akkermansia muciniphila may be an evidence-based treatment plan for sunitinib-induced diarrhea. Elimination of potentially pathogenic species such as Bacteroides may reduce the side effects of sunitinib, thereby improving the prognosis of patients. Next, we will conduct further studies required to explore the mechanism of intestinal flora affecting sunitinib associated diarrhea.

\section{Acknowledgments}

We thank Fuyu Chen for excellent assistant in statistical assistance and constructive advice.

\section{Funding}

This work was supported by the Natural Science Foundation of China (Nos. 81302067 and 81502360), the Natural Science Foundation of Fujian Province (Nos. 2016J01576, 2020J011147 and 2021J01281), and the Science and Technology Innovation Joint Foundation of Fujian Province (No.2017Y9125).

\section{Disclosure}

The authors report no conflicts of interest in this work.

\section{References}

1. Siegel RL, Miller KD, Jemal A. Cancer statistics, 2017. CA Cancer J Clin. 2017;67(1):7-30. doi:10.3322/caac.21387

2. Ravaud A, Motzer RJ, Pandha HS, et al. Adjuvant sunitinib in high-risk renal-cell carcinoma after nephrectomy. $N$ Engl $J$ Med. 2016;375(23):2246-2254. doi:10.1056/NEJMoa1611406

3. Escudier B, Porta C, Schmidinger M, et al. Renal cell carcinoma: ESMO clinical practice guidelines for diagnosis, treatment and follow-up. Ann Oncol. 2016;27(suppl 5):v58-58v68. doi:10.1093/ annonc/mdw328

4. Rini BI, Escudier B, Tomczak P, et al. Comparative effectiveness of axitinib versus sorafenib in advanced renal cell carcinoma (AXIS): a randomised Phase 3 trial. Lancet. 2011;378(9807):1931-1939. doi:10.1016/S0140-6736(11)61613-9

5. Sh Z. Adjuvant Sunitinib in Renal-Cell Carcinoma. $N$ Engl J Med. 2017;376(9):893.

6. Motzer RJ, Hutson TE, Tomczak P, et al. Sunitinib versus interferon alfa in metastatic renal-cell carcinoma. $N$ Engl J Med. 2007;356 (2):115-124. doi:10.1056/NEJMoa065044

7. Sternberg CN, Davis ID, Mardiak J, et al. Pazopanib in locally advanced or metastatic renal cell carcinoma: results of a randomized Phase III trial. J Clin Oncol. 2010;28(6):1061-1068. doi:10.1200/JCO.2009.23.9764

8. Schmidinger M. Understanding and managing toxicities of vascular endothelial growth factor (VEGF) inhibitors. EJC Suppl. 2013;11 (2):172-191. doi:10.1016/j.ejcsup.2013.07.016

9. Appleby L, Morrissey S, Bellmunt J, Rosenberg J. Management of treatment-related toxicity with targeted therapies for renal cell carcinoma: evidence-based practice and best practices. Hematol Oncol Clin North Am. 2011;25(4):893-915. doi:10.1016/j.hoc.2011.05.004

10. Dzutsev A, Goldszmid RS, Viaud S, Zitvogel L, Trinchieri G. The role of the microbiota in inflammation, carcinogenesis, and cancer therapy. Eur $J$ Immunol. 2015;45(1):17-31. doi:10.1002/ eji.201444972

11. De Santis S, Cavalcanti E, Mastronardi M, Jirillo E, Chieppa M. Nutritional keys for intestinal barrier modulation. Front Immunol. 2015;6:612. doi:10.3389/fimmu.2015.00612

12. Vaishnava S, Behrendt CL, Ismail AS, Eckmann L, Hooper LV. Paneth cells directly sense gut commensals and maintain homeostasis at the intestinal host-microbial interface. Proc Natl Acad Sci U S A. 2008;105(52):20858-20863. doi:10.1073/pnas.0808723105

13. Peterson LW, Artis D. Intestinal epithelial cells: regulators of barrier function and immune homeostasis. Nat Rev Immunol. 2014;14 (3):141-153. doi:10.1038/nri3608

14. Janelsins BM, Lu M, Datta SK. Altered inactivation of commensal LPS due to acyloxyacyl hydrolase deficiency in colonic dendritic cells impairs mucosal Th17 immunity. Proc Natl Acad Sci U S A. 2014;111(1):373-378. doi:10.1073/pnas.1311987111

15. Park JH, Jeong SY, Choi AJ, Kim SJ. Lipopolysaccharide directly stimulates Th17 differentiation in vitro modulating phosphorylation of RelB and NF-kB1. Immunol Lett. 2015;165(1):10-19. doi:10.1016/j.imlet.2015.03.003

16. Roy S, Trinchieri G. Microbiota: a key orchestrator of cancer therapy. Nat Rev Cancer. 2017;17(5):271-285. doi:10.1038/nrc.2017.13

17. Fan L, Iseki S. Immunohistochemical localization of vascular endothelial growth factor in the endocrine glands of the rat. Arch Histol Cytol. 1998;61(1):17-28. doi:10.1679/aohc.61.17

18. Chowdhury N, Drake CG. Kidney cancer: an overview of current therapeutic approaches. Urol Clin North Am. 2020;47(4):419-431. doi:10.1016/j.ucl.2020.07.009 
19. Rizzo A, Mollica V, Santoni M, Massari F. Risk of selected gastrointestinal toxicities in metastatic renal cell carcinoma patients treated with immuno-TKI combinations: a meta-analysis. Expert Rev Gastroenterol Hepatol. 2021;15(10):1225-1232. doi:10.1080/ 17474124.2021.1948328

20. Pal SK, Li SM, Wu X, et al. Stool bacteriomic profiling in patients with metastatic renal cell carcinoma receiving vascular endothelial growth factor-tyrosine kinase inhibitors. Clin Cancer Res. 2015;21 (23):5286-5293. doi:10.1158/1078-0432.CCR-15-0724

21. Caporaso JG, Kuczynski J, Stombaugh J, et al. QIIME allows analysis of high-throughput community sequencing data. Nat Methods. 2010;7(5):335-336. doi:10.1038/nmeth.f.303

22. Edgar RC. UPARSE: highly accurate OTU sequences from microbial amplicon reads. Nat Methods. 2013;10(10):996-998. doi:10.1038/ nmeth.2604

23. Amato KR, Yeoman CJ, Kent A, et al. Habitat degradation impacts black howler monkey (Alouatta pigra) gastrointestinal microbiomes. ISME J. 2013;7(7):1344-1353. doi:10.1038/ismej.2013.16

24. Wang Y, Sheng HF, He Y, et al. Comparison of the levels of bacterial diversity in freshwater, intertidal wetland, and marine sediments by using millions of illumina tags. Appl Environ Microbiol. 2012;78 (23):8264-8271. doi:10.1128/AEM.01821-12

25. Bates ST, Clemente JC, Flores GE, et al. Global biogeography of highly diverse protistan communities in soil. ISME J. 2013;7 (3):652-659. doi:10.1038/ismej.2012.147

26. Jiang XT, Peng X, Deng GH, et al. Illumina sequencing of $16 \mathrm{~S}$ rRNA tag revealed spatial variations of bacterial communities in a mangrove wetland. Microb Ecol. 2013;66(1):96-104. doi:10.1007/ s00248-013-0238-8

27. Oberauner L, Zachow C, Lackner S, Högenauer C, Smolle KH, Berg G. The ignored diversity: complex bacterial communities in intensive care units revealed by $16 \mathrm{~S}$ pyrosequencing. Sci Rep. 2013;3:1413. doi:10.1038/srep01413

28. Zhang C, Li S, Yang L, et al. Structural modulation of gut microbiota in life-long calorie-restricted mice. Nat Commun. 2013;4:2163. doi: $10.1038 /$ ncomms3163

29. Liu J, Nicum S, Reichardt P, et al. Assessment and management of diarrhea following VEGF receptor TKI treatment in patients with ovarian cancer. Gynecol Oncol. 2018;150(1):173-179. doi:10.1016/ j.ygyno.2018.03.058

30. Armani RG, Ramezani A, Yasir A, Sharama S, Canziani M, Raj DS. Gut microbiome in chronic kidney disease. Curr Hypertens Rep. 2017;19(4):29.

31. Greenblum S, Turnbaugh PJ, Borenstein E. Metagenomic systems biology of the human gut microbiome reveals topological shifts associated with obesity and inflammatory bowel disease. Proc Natl Acad Sci U S A. 2012;109(2):594-599. doi:10.1073/pnas.1116053109

32. Jie Z, Xia H, Zhong SL, et al. The gut microbiome in atherosclerotic cardiovascular disease. Nat Commun. 2017;8(1):845. doi:10.1038/ s41467-017-00900-1

33. Qin J, Li Y, Cai Z, et al. A metagenome-wide association study of gut microbiota in type 2 diabetes. Nature. 2012;490(7418):55-60. doi:10.1038/nature11450
34. Qin N, Yang F, Li A, et al. Alterations of the human gut microbiome in liver cirrhosis. Nature. 2014;513(7516):59-64. doi:10.1038/ nature 13568

35. Hooper LV, Xu J, Falk PG, Midtvedt T, Gordon JI. A molecular sensor that allows a gut commensal to control its nutrient foundation in a competitive ecosystem. Proc Natl Acad Sci U S A. 1999;96 (17):9833-9838. doi:10.1073/pnas.96.17.9833

36. Louis P, Flint HJ. Diversity, metabolism and microbial ecology of butyrate-producing bacteria from the human large intestine. FEMS Microbiol Lett. 2009;294(1):1-8. doi:10.1111/j.15746968.2009.01514.x

37. Bhatt AP, Redinbo MR, Bultman SJ. The role of the microbiome in cancer development and therapy. CA Cancer J Clin. 2017;67 (4):326-344. doi:10.3322/caac. 21398

38. Wang Q, Garrity GM, Tiedje JM, Cole JR. Naive Bayesian classifier for rapid assignment of rRNA sequences into the new bacterial taxonomy. Appl Environ Microbiol. 2007;73(16):5261-5267. doi:10.1128/AEM.00062-07

39. Tu P, Bian X, Chi L, et al. Characterization of the functional changes in mouse gut microbiome associated with increased akkermansia muciniphila population modulated by dietary black raspberries. ACS Omega. 2018;3(9):10927-10937. doi:10.1021/acsomega.8b00064

40. Massari F, Rizzo A, Mollica V, et al. Immune-based combinations for the treatment of metastatic renal cell carcinoma: a meta-analysis of randomised clinical trials. Eur J Cancer. 2021;154:120-127. doi:10.1016/j.ejca.2021.06.015

41. Salgia NJ, Bergerot PG, Maia MC, et al. Stool microbiome profiling of patients with metastatic renal cell carcinoma receiving anti-PD-1 immune checkpoint inhibitors. Eur Urol. 2020;78(4):498-502. doi:10.1016/j.eururo.2020.07.011

42. Mi H, Dong Y, Zhang B, et al. Bifidobacterium infantis ameliorates chemotherapy-induced intestinal mucositis via regulating $\mathrm{T}$ cell immunity in colorectal cancer rats. Cell Physiol Biochem. 2017;42 (6):2330-2341. doi:10.1159/000480005

43. Chang CW, Liu CY, Lee $\mathrm{HC}$, et al. Lactobacillus casei variety rhamnosus probiotic preventively attenuates 5-fluorouracil/oxaliplatin-induced intestinal injury in a syngeneic colorectal cancer model. Front Microbiol. 2018;9:983. doi:10.3389/fmicb.2018.00983

44. Ganji-Arjenaki M, Rafieian-Kopaei M. Probiotics are a good choice in remission of inflammatory bowel diseases: a meta analysis and systematic review. $J$ Cell Physiol. 2018;233(3):2091-2103. doi:10.1002/jcp.25911

45. Mego M, Chovanec J, Vochyanova-Andrezalova I, et al. Prevention of irinotecan induced diarrhea by probiotics: a randomized double blind, placebo controlled pilot study. Complement Ther Med. 2015;23 (3):356-362. doi:10.1016/j.ctim.2015.03.008

46. Furusawa Y, Obata Y, Fukuda S, et al. Commensal microbe-derived butyrate induces the differentiation of colonic regulatory $\mathrm{T}$ cells. Nature. 2013;504(7480):446-450. doi:10.1038/nature12721

\section{Publish your work in this journal}

Cancer Management and Research is an international, peer-reviewed open access journal focusing on cancer research and the optimal use of preventative and integrated treatment interventions to achieve improved outcomes, enhanced survival and quality of life for the cancer patient.
The manuscript management system is completely online and includes a very quick and fair peer-review system, which is all easy to use. Visit http://www.dovepress.com/testimonials.php to read real quotes from published authors. 